\title{
Percepciones y prácticas socio-ambientales en las comunidades aledañas al Corredor
}

\section{Biológico Montes del Aguacate}

\author{
Socio-environmental perceptions and practices in the communities surrounding the Organic \\ Corridor Montes del Aguacate
}

Percepções e práticas socioambientais nas comunidades do entorno do Corredor Biológico Montes

del Aguacate

Recibido: 06/05/2021 | Revisado: 10/05/2021 | Acepto: 15/05/2021 | Publicado: 30/05/2021

\author{
Marielos Vargas Morales \\ ORCID https://orcid.org/0000-0001-9307-4946 \\ Universidad Nacional de Costa Rica, Costa Rica \\ E-mail marielos.vargas.morales@una.ac
}

\begin{abstract}
Resumen
El Corredor Biológico Montes del Aguacate de Costa Rica en una zona de conectividad donde se genera una estrecha relación entre las poblaciones humanas y el entorno, lo cual impacta directamente los ecosistemas naturales. El estudio socio-ambiental se construye desde la mirada de sus pobladores por lo que se aplica una encuesta de percepción con preguntas abiertas y cerradas a 128 personas en 30 distritos donde se ubica el corredor biológico. Las categorías de análisis versan sobre: 1) uso del suelo y actividades productivas sostenibles; 2) organización local y capacitación en temas ambientales y 3) prácticas tendientes a la protección del ambiente. Se identifica en el corredor biológico dos actividades productivas relevantes; el cultivo de café y la ganadería, lo cual moviliza la economía local, pero a la vez, genera una significativa tala de árboles y algunas acciones contaminantes. En la población participante existe desconocimiento generalizado sobre la coexistencia con el corredor biológico. Prevalece el discurso de cuidar el entorno natural, pero no se acompaña con acciones concretas. El desarrollo de la zona tiene un fuerte ligamen con el turismo rural, donde se aprovecha la belleza escénica y la biodiversidad natural. Se rescata el reconocimiento comunitario a las instancias administradoras del agua; la concientización de algunos pobladores con acciones en pro de un ambiente sostenible y el poder que como colectivo han redescubierto algunas comunidades en la protección del entorno natural.
\end{abstract}

Palabras clave: Corredor biológico; Conservación; Problemas socio-ambientales; Prácticas sostenibles; Sostenibilidad ambiental.

\begin{abstract}
The Biological Corridor Montes del Aguacate in Costa Rica is a connectivity zone where a close relationship between human populations and the environment is generated, which directly impacts natural ecosystems. The socioenvironmental study is built from the viewpoint of its inhabitants so a perception survey is applied with open and closed questions to 128 people in 30 districts where the biological corridor is located. The categories of analysis deal with: 1) land use and sustainable productive activities; 2) local organization and training in environmental issues; and 3) practices aimed at environmental protection. Two relevant productive activities are identified in the biological corridor: coffee cultivation and cattle raising, which mobilize the local economy, but at the same time, generate significant tree felling and some polluting actions. There is a general lack of knowledge among the participating population about the coexistence with the biological corridor. The discourse of caring for the natural environment prevails, but is not accompanied by concrete actions. The development of the area is strongly linked to rural tourism, where the scenic beauty and natural biodiversity are taken advantage of. Community recognition of the water management authorities, the awareness of some inhabitants with actions for a sustainable environment and the power that some communities have rediscovered as a collective in the protection of the natural environment are rescued.

Keywords: Biological corridor; Conservation; Socio-environmental; Problems; Sustainable practices; Environmental sustainability.

\section{Resumo}

O Corredor Biológico Montes del Aguacate na Costa Rica é uma zona de conectividade onde se gera uma estreita relação entre as populações humanas e o meio ambiente, o que impacta diretamente os ecossistemas naturais. $\mathrm{O}$ estudo socioambiental é construído na perspectiva de seus moradores, de forma que uma pesquisa de percepção com questões abertas e fechadas é aplicada a 128 pessoas em 30 bairros onde está localizado o corredor biológico. As
\end{abstract}


categorias de análise tratam de: 1) uso da terra e atividades produtivas sustentáveis; 2) organização local e treinamento em questões ambientais e 3) práticas voltadas para a proteção do meio ambiente. Duas atividades produtivas relevantes são identificadas no corredor biológico; o cultivo do café e da pecuária, que mobiliza a economia local, mas ao mesmo tempo, gera um significativo corte de árvores e algumas ações poluidoras. Na população participante há um desconhecimento geral sobre a convivência com o corredor biológico. O discurso do cuidado com o meio ambiente prevalece, mas não é acompanhado de ações concretas. $\mathrm{O}$ desenvolvimento da zona tem uma forte ligação com o turismo rural, onde se aproveita a beleza paisagística e a biodiversidade natural. O reconhecimento da comunidade aos órgãos de administração de água é resgatado; a conscientização de alguns moradores com ações em prol de um meio ambiente sustentável e o poder que, como grupo, algumas comunidades redescobriram na proteção do meio ambiente natural.

Palavras-chave: Corredor biológico; Conservação; Problemas socioambientais; Práticas sustentáveis; Sustentabilidade ambiental.

\section{Introducción}

De acuerdo con el Estado de la Biodiversidad Costa Rica 2014-2018, el país supera el 6\% de la biodiversidad mundial. Por las políticas establecidas y los esfuerzos generados hacia la protección de los recursos del medio ambiente, este se considera un país ecológico. Sin embargo, existe un desfase entre el crecimiento económico y la sostenibilidad que debe acompañar de manera paralela el desarrollo (Estado de la Nación, 2014, p. 137). No obstante, se rescatan las iniciativas desarrolladas en materia ambiental bajo la figura del Ministerio de Ambiente y Energía (MINAE), ya que a la fecha se cuenta con 11 áreas de conservación como estrategias para la protección de los recursos ambientales. De acuerdo con el Sistema Nacional de Áreas de Conservación (SINAC) (2011), estas "procuran mantener los procesos ecológicos, científicamente identificados" (p. 12), "como una estrategia para disminuir el efecto de los procesos de fragmentación en Costa Rica" (Sandoval y Castillo, 2011, p. 21). Las áreas de conservación contienen otras figuras reguladas jurídicamente para la protección de zonas específicas como: parques nacionales, corredores biológicos, reservas forestales, reservas biológicas, zonas protectoras, refugios de vida silvestre, humedales y reservas naturales absolutas (Sandoval y Castillo, 2011, p. 22).

Costa Rica tiene 45 corredores biológicos definidos; estos territorios son regulados por el SINAC, y se adscriben al Programa Nacional de Corredores Biológicos; iniciativa creada mediante Decreto Ejecutivo No 33106-MINAE, con el objetivo de "promocionar la conservación y uso sostenible de la biodiversidad en Costa Rica desde una perspectiva de conectividad eco-sistémica funcional y estructural (MINAE, 2016). Estas áreas ocupan aproximadamente el 33.1\% del territorio nacional (SINAC 2018, p. 17) y se resguardan mediante la Política Nacional de Biodiversidad de Costa Rica 2015-2030 y la Ley de Biodiversidad, decreto $N^{o}$ 34433-MINAE; entre otras leyes conexas.

El presente artículo socializa el trabajo desarrollado en el Corredor Biológico Montes del Aguacate (CBMA), el cual se realiza en el marco del Proyecto denominado "Análisis de los Corredores Biológicos como estrategia para la conservación y el desarrollo local en Costa Rica”. El artículo comparte acciones tendientes al cumplimiento del tercer objetivo del proyecto, cuya finalidad es caracterizar comunidades humanas dentro del Corredor Biológico Montes del Aguacate, en aspectos socioambientales para la valoración del conocimiento en relación con el uso sustentable de los recursos del entorno (Universidad Nacional de Costa Rica, 2017).

El CBMA fue creado en el año 2001; es un área de conectividad entre paisajes naturales y modificados por el ser humano. Se encuentra en el Área de Conservación Cordillera Volcánica Central y Pacífico Central de Costa Rica. Es una zona rica en biodiversidad con gran cantidad de comunidades aledañas, entorno donde sus pobladores realizan diversidad de actividades productivas y de subsistencia; relación que modifica el territorio e inevitablemente trastoca las condiciones requeridas para el desarrollo natural de los ecosistemas (Solano, 2017).

Los corredores biológicos son áreas silvestres protegidas con miras al mantenimiento de la biodiversidad y la conservación de los recursos naturales (MINAE, 2016). Estos espacios protegen las especies y favorecen la evolución de los ecosistemas (Solano, 2017). Así mismo, ayudan a "establecer la conectividad estructural mediante enlaces físicos biológicos- 
ecológicos entre áreas o hábitats para garantizar conectividad funcional de la fauna y la flora, en cuanto a la movilidad de especies y dispersión genética (Schlönvoigt, 2012, p.16). Los ecosistemas presentes en estos espacios biológicos permiten que diversidad de organismos interactúen en un medio físico específico (Armenteras, González, Vergara, Luque, Rodríguez y Bonilla, 2016). Por ello, la protección de los hábitats a través de los corredores biológicos "es la mejor manera de conservar la diversidad biológica, ya que al proteger su ambiente de vida se busca generar las condiciones para su supervivencia" (Sánchez, 2002, p. 234).

La protección de los ecosistemas en los corredores biológicos "ejercen diversas funciones tanto ecológicas como económicas a saber: la protección de los recursos hídricos, el control de la erosión, la regulación del ciclo hidrológico y la provisión de abrigo para la fauna" (Sánchez, 2002, p. 224).

La conservación y manejo de la biodiversidad requiere el conocimiento de los mecanismos y procesos ecológicos, evolutivos y conductuales que generan, estructuran, y mantienen la diversidad biológica a varias escalas y en diferentes niveles de organización, desde genes y poblaciones hasta las comunidades, ecosistemas y paisajes, de cómo estos resultan de las interacciones de los organismos con su entorno abiótico y biótico, y de cómo estos responden a los cambios globales. (Cuenca, Guevara y Franco, 2018, p. 72-73)

De acuerdo con lo expuesto, la protección de los ecosistemas requiere estudios donde se devele las situaciones que subyacen en estas zonas boscosas fragmentadas, donde se desarrolla la vida social y productiva de poblaciones humanas, accionar que modifica los hábitats y alteran los ecosistemas, lo cual, es una de las principales causas que lleva a la pérdida de la biodiversidad natural. En este sentido, existe el "reto de integrar las comunidades con su entorno de manera armoniosa" (Calderón, 2019, p.28). Ante estas relaciones y el deterioro del ambiente, es importante generar investigaciones que permitan tener una "aproximación en temas relacionadas con la caracterización de los componentes en los niveles de especies, recursos genéticos, poblaciones, comunidades y ecosistemas" (Cuenca, Guevara y Franco, 2018, p. 71). Precisamente, la relación estrecha entre las comunidades aledañas con una zona de conservación natural, evidencia impactos profundos que generan problemas socio-ambientales (Bennett, 2003).

Desde la perspectiva de Aliste y Rabi (2012), lo socio-ambiental vincula la sociedad con el medio ambiente y estos problemas inician con las demandas de la sociedad cuando estas tienden a una degradación ambiental. De acuerdo con Morales, Aliste, Neira y Urquiza (2019) una dificultad que suma en la comprensión de los problemas socio-ambientales es la acepción polisémica del significado que le asignan los diversos actores, donde las percepciones juegan un papel importante para comprender la realidad y el accionar de las personas con su medio natural.

Las percepciones son una construcción social, mediada por la cultura. De manera general, las personas asumen la cultura y modos de vida a partir de lo que la sociedad como colectivo construye. Según Vargas (1994) la construcción de las percepciones implica una interacción constante entre el individuo y lo que la sociedad le ofrece, de manera que dicha construcción, si bien es individual, está mediada por lo que dicta el colectivo social. En esta correspondencia las personas desarrollan prácticas cotidianas concatenadas con lo que piensan y consideran adecuado; y estas a su vez, están mediadas por sus percepciones (Álvarez, 2010).

Las percepciones tanto en su aprehensión como lo que se derivan de ellas, están impregnadas por los valores construidos como sociedad, el cómo actuamos presenta esta relación directa. Por tanto, para actuar en la línea de la sostenibilidad necesariamente aplicamos valores intrínsecos que surgen de nuestras percepciones y concepciones (Zarta, 2018). Haciendo un ligamen, entre las percepciones y prácticas sostenibles, el enfoque en sostenibilidad ambiental implica cambios perceptuales, conductuales y culturales ya que se obtendrá siempre y cuando "la explotación de los recursos naturales se 
mantenga dentro de los límites de la regeneración y el crecimiento natural" (Zarta, 2018, p. 218). Al respecto, entender cómo la pérdida y fragmentación del hábitat, es una situación que se deriva de las intervenciones humanas, es una prioridad.

De acuerdo con lo expuesto, es necesario el análisis de las percepciones y prácticas de las personas en relación con el entorno, lo cual brinde insumos para generar propuestas que aminoren los estragos que el ser humano causa en el medio ambiente (Zarta, 2018). Por ello, el presente artículo busca un acercamiento a las percepciones, interacción y prácticas que se genera en el CBMA en torno al desarrollo sostenible, lo cual pueda brindar una mirada de la realidad socio-ambiental presente, cuyos insumos puedan fundamentar propuestas tendientes a la conservación y protección de los diversos ecosistemas que coexisten en el corredor biológico.

\section{Metodología}

La construcción metodológica se sustenta en la revisión de la literatura, la cual brinda insumos para el planteamiento coherente del estudio, el cual versa sobre las percepciones de las personas. De acuerdo con Vargas (1994), estas refieren a un proceso biocultural que se construye desde la infancia, lo cual implica recepción y respuesta a estímulos, pero paralelamente interpretaciones impregnadas por la cultura y la ideología imperante. Por tanto, la investigación se enmarca en el paradigma interpretativo, ya que a través de este se busca comprender la realidad desde la perspectiva significativa de quienes la viven y piensan (Fernández, Hernández y Baptista, 2014).

Desde el posicionamiento teórico del paradigma interpretativo se busca "encontrar sentido a los fenómenos en función de los significados que las personas les otorguen” (Fernández, Hernández y Baptista (2014, p. 9). Con este fundamento se plantea de manera coherente la pregunta de investigación ¿Cuáles son las percepciones y prácticas socio-ambientales de las poblaciones aledañas al Corredor Biológico Montes del Aguacate? En esta misma lógica, se asume el enfoque cualitativo, por tanto, el proceso indagatorio y de análisis "se mueve de manera dinámica en dos sentidos: entre los hechos y su interpretación" (Fernández, Hernández, Baptista, 2014, p.7). A la vez, se enmarca dentro de los estudios descriptivos donde se busca caracterizar las poblaciones aledañas respecto a su relación con el entorno ambiental (Hernández, Fernández y Baptista, 2014).

Desde el encuadre cualitativo se realizan varias técnicas: un grupo focal con organismos locales (Asociación de Desarrollo, Junta de Educación, Patronato Escolar, Comités Comunitarios) y se aplican 6 entrevistas en profundidad a organizaciones que desarrollan proyectos locales. Si bien, el estudio es cualitativo, se aborda la encuesta (E) como técnica cuantitativa y principal de la investigación. Esta se aplica a una muestra estadística con $85 \%$ de confiabilidad y $5 \%$ como margen de error. La encuesta se aplica a 128 personas de 30 distritos ubicados en el CBMA. La encuesta se valida aplicando criterio de expertos para los fines requeridos y se titula "Percepción de los pobladores de comunidades aledañas al Corredor Biológico Montes del Aguacate" e incluye preguntas abiertas y cerradas. Los tópicos que se plantean en la encuesta están concatenados con la pregunta de investigación y sobre estos, versa el análisis: a- uso del suelo y actividades productivas sostenibles; b- organización local y capacitación en temas ambientales y; c-prácticas tendientes a la protección del ambiente. La información obtenida se contrasta con datos que se derivan de las técnicas cualitativas, con la intencionalidad de profundizar en la realidad que subyace en el CBMA.

El análisis de los resultados se realiza con sustento en el desarrollo sostenible, considerando que el desarrollo solo se logra con el éxito en tres elementos interactuantes y correlacionados: lo económico, lo social y lo ambiental (Zarta, 2018). Al respecto, en Costa Rica se creó una metodología para evaluar la efectividad en el manejo de los corredores biológicos, donde se abordan las dimensiones: ecológica, gestión y socioeconómica. Precisamente en el estudio se asume la tercera dimensión, ya que esta plantea la relación existente entre las comunidades aledañas con el corredor biológico. Desde esta perspectiva, se 
busca valorar el uso sustentable de los recursos, lo cual pueda contribuir con el mejoramiento de las condiciones de vida de sus pobladores (Canet, Finegan y Herrera, 2012).

El estudio se realiza entre mayo de 2018 a junio de 2020, pero se enmarca en una investigación que finaliza en el 2021, por lo que el documento socializa un avance de los resultados. Para la selección de la zona de estudio, se aplica el criterio de constitución jurídica, el cual consiste en que el corredor biológico estuviera delimitado por el Estado, haber sido juramentado por el Sistema Nacional de Áreas Protegidas y poseer Consejo Local.

El área de estudio está conformada por 55 219,56 hectáreas, se ubica casi en su totalidad en la provincia de Alajuela, con una extensión de 34 805,9 ha (99.63\%) y en Puntarenas la cobertura es de 201,1904 (0.36\%). Comprende 8 cantones: Atenas, Orotina, San Mateo, Naranjo, San Ramón, Naranjo, Montes de Oro y Esparza. (SINAC, 2015). El CBMA favorece la conectividad entre diferentes Áreas Silvestres Protegidas (ASP). Este inicia “en la Reserva Biológica Alberto Manuel Brenes, incluye el Refugio Nacional de Vida Silvestre Mixto Peñas Blancas y las Zonas Protectoras Monte de Oro, Cerros Atenas, Río Grande de Atenas y Cerro El Chompipe" (SINAC, 2015, p. 5).

\section{Resultados}

\subsection{Uso del suelo y actividades productivas sostenibles}

El 48\% de las personas encuestadas indican que el suelo se destina a cultivos permanentes, donde figura el cultivo del café. Sin embargo, se percibe reducción en su siembra y aunque se considera que existe "una mejoría en cuanto al tratamiento de los suelos para el cultivo, se sigue contaminando con los beneficios de café" (E 29). Se advierte que los avances que mencionan refiere en cómo se debe hacer la siembra para que el suelo no se erosione y no a los productos que utilizan en el cultivo.

De acuerdo con los datos que se muestran en la Figura 1, el 24\% de los encuestados expresa que el terreno se destina a pastos. Se identifican cambios en la estructura del paisaje (carreteras, urbanizaciones, mueblerías y comercio), estas modificaciones se asocian mayormente con la tala de árboles. Además, se registra la necesidad de mayor siembra de árboles en cafetales, pastizales y cerca de las fuentes de agua. El $18.7 \%$ de los encuestados considera que se ha dado un aumento en la apertura de polleras, en menor escala chancheras $(5.4 \%)$ y luego viveros $(3.9 \%)$.

Figura 1. Percepción sobre el uso del suelo por parte de encuestados en el Corredor Biológico Montes del Aguacate.

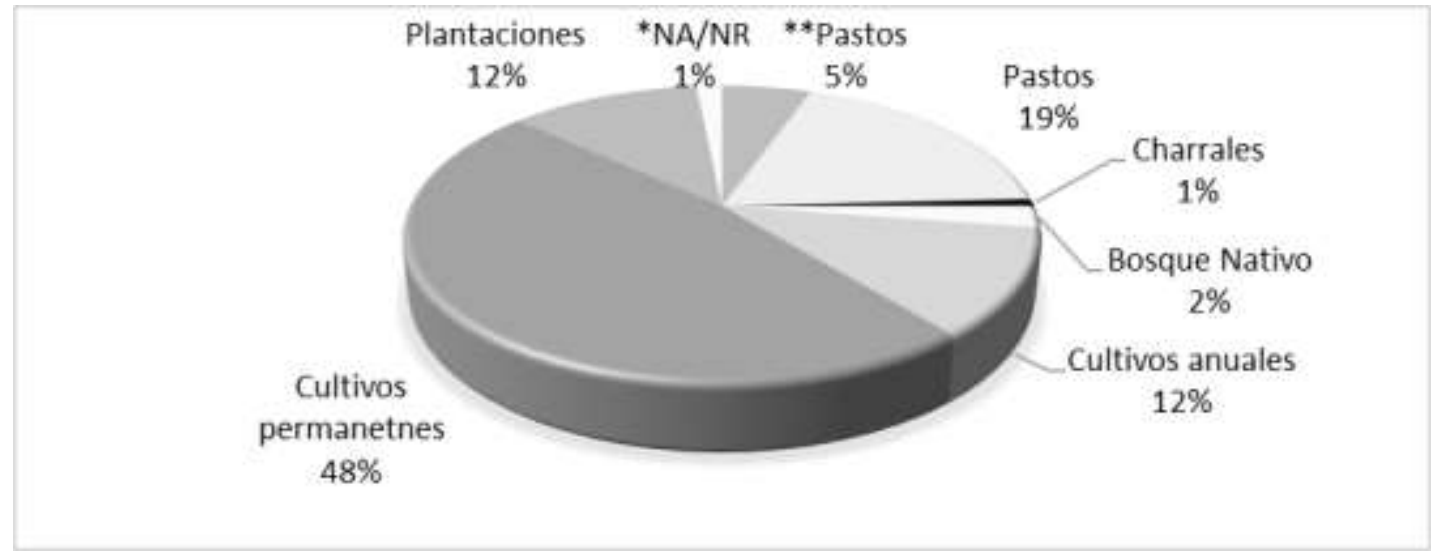

*NS/NR: no sabe o no responder. ** Pastos sin árboles dispersos. Fuente: Autores. 


\subsection{Organización local y capacitación en temas ambientales}

Como se observa en la Figura 2, se identifica que un $1 \%$ de las personas consultadas participan en grupos que atiendan el tema ambiental; así mismo, se registra la carencia en capacitación sobre temas ambientes. El $23 \%$ forma parte de grupos orientados al desarrollo local (Asociaciones de Desarrollo y Asociaciones Administradoras de Sistemas de Acueductos y Alcantarillados Sanitarios). Un 11\% conforman grupos educativos (Juntas de Educación y Patronato Escolar). Solo el 20\% indica haber recibido algún tipo de capacitación sobre temas relacionados con el ambiente, donde figura el reciclaje.

Figura 2. Porcentaje según tipo de agrupación local de encuestados en el Corredor Biológico Montes del Aguacate.

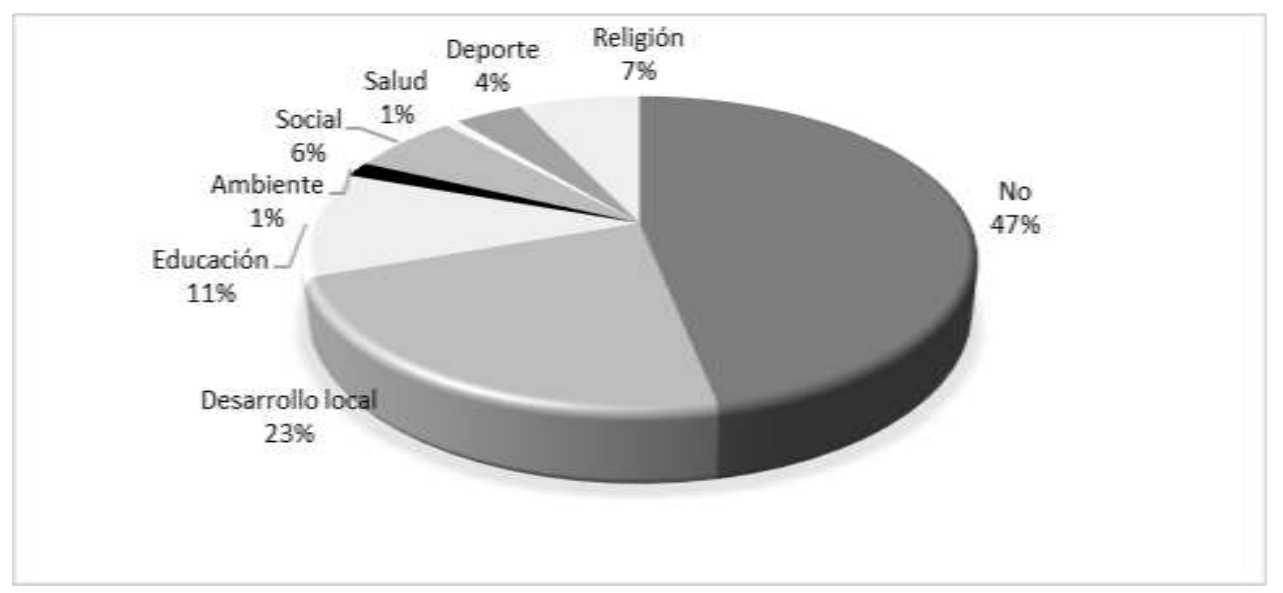

Fuente: Autores.

Se rescata la figura de las Asociaciones Administradoras de Sistemas de Acueductos y Alcantarillados Sanitarios (ASADAS) como responsables del cuido del agua, a las cuáles se les brinda reconocimiento por la calidad del agua y la labor que desempeñan en las comunidades. Es importante acotar, que se identifica percepciones sobre la disminución del líquido en fuentes agua y el experimentar veranos extensos.

En el CBMA se visualiza una serie de acciones comerciales y turísticas donde se aprovecha la riqueza en biodiversidad, la belleza escénica, el aventurismo y la degustación de comida tradicional lugareña. Precisamente, la Red de Reservas Naturales del Corredor Biológico Montes del Aguacate integra esfuerzos locales, no gubernamentales y participación estatal para el desarrollo del turismo rural, lo que ha promovido el auge de servicios en la zona y la movilización de la economía comunitaria (EP-3).

\subsection{Prácticas tendientes a la protección del ambiente}

Tal y como se muestra en la Tabla 1, se identifica que el 93\% de las personas encuestadas desconoce que vive en una comunidad aledaña al CBMA. El 51\% asegura tener algún tipo de práctica positiva, donde la acción que mayormente reportan es el reciclaje (26\%); seguido del cuido del suelo (24\%) que refiere mayormente en esparcir desechos orgánicos en el jardín.

De acuerdo con el $43 \%$ de los encuestados existe mayor deforestación, contaminación y reducción de la biodiversidad en el corredor biológico; así como la percepción que las personas hablan de cuidar el ambiente, pero realmente no realizan prácticas que sean coherentes con este enfoque (E-6). 
Tabla 1. Aspectos de consulta en pobladores del Corredor Biológico Montes del Aguacate,

\begin{tabular}{|l|l|l|}
\hline \multicolumn{1}{|c|}{ Indicadores } & Sí & No \\
\hline $\begin{array}{l}\text { 1. } \\
\text { Conocimiento sobre la ubicación de la comunidad en el entorno del corredor } \\
\text { biológico }\end{array}$ & $7 \%$ & $93 \%$ \\
\hline 2. Noción sobre lo que es un corredor biológico* & $14 \%$ & 82 \\
\hline 3. Observación de cambios ambientales & $55 \%$ & $45 \%$ \\
\hline 4. Prácticas orientadas a la protección del ambiente & $51 \%$ & $49 \%$ \\
\hline
\end{tabular}

*4\%: No sabe/No responde. Fuente: Autores.

Al respecto, algunos encuestados indican acciones orientadas al cuido del ambiente, pero estas no corresponden. Ejemplo de ello, quemar basura, enterrarla; o bien, depositar los desechos en el carro recolector considerando esta acción como reciclaje, sin realizar el proceso de clasificación y separación, entre otros. Existe reporte de afectación con desechos sólidos, aguas negras y químicos que van a dar a algunas las fuentes de agua. Algunos pobladores lavan los equipos para fumigar en las quebradas y utilizan el mismo equipo para abonar y esparcir herbicidas (EP-3).

Se identifica dos comunidades que gestionaron el cierre de una chanchera y una pollera, pero persiste molestia ante otras chancheras por el mal olor y los materiales que van a dar a los ríos (E-91), además porque las denuncias legales no fructifican (E-98).

\section{Discusión}

El desarrollo sostenible tiene que ver con el manejo ambiental armonioso de las fuentes de producción y su relación con el impacto laboral, económico y social (Miranda, 2013, p. 95). En relación con las actividades socio-productivas en la zona del CBMA, existe una marcada tendencia al cultivo del café y la ganadería. Precisamente, el café ha sido uno de los pilares de la economía costarricense y base del desarrollo social y cultural del país. En el año 2016, aportó un 6,04\% del Producto Interno Bruto Agropecuario. Sin embargo, en el CBMA se percibe la reducción de este cultivo y coincide con lo acaecido en el ámbito nacional en el periodo entre 1994-95 y 2016-17, donde se registra una reducción del 17.7\% (Instituto del Café de Costa Rica, 2017). Si bien, es importante que el Estado revitalice la siembra del café, por los aportes que tiene en el desarrollo nacional y local; es necesario que su promoción se realice en el marco de un desarrollo sostenible; donde se articule la sostenibilidad económica con la ambiental (Zarta 2018).

En el CBMA existe la percepción que aún se genera contaminación con los beneficios de café. Cuando no se hace un tratamiento adecuado en la producción del cultivo, este puede ocasionar también el problema de la contaminación con las aguas mieles, lo cual debe ser entendido como un "conflicto de carácter ambiental” (Montero y Sandí, 2009). Los residuos que se generan van a dar al suelo y por el proceso de absorción natural, pueden llegar a afectar las fuentes de agua subterránea, y esto a su vez, contaminar las nacientes de donde se toma el líquido para consumo humano.

Existen estudios que develan consecuencias generadas por la contaminación del agua a partir de los residuos del café. En el 2008, se realizó un estudio en una planta procesadora del café en Zimma una zona de Etiopía. En la investigación se advierte contaminación de las aguas residuales. Las poblaciones aledañas que consumían el agua contaminada, sufrieron afectación en la salud vivenciando molestias como: irritación de ojos, oídos y piel, dolor de estómago, náuseas y problemas respiratorios (Haddis \& Devi, 2008). 
Con la producción del café es comprensible que se busque eficiencia en las prácticas y es común que los cultivos sufran amenazas de enfermedades que deben ser controladas mediante plaguicidas. Según la Coordinadora Latinoamericana y del Caribe de Pequeños Productores y Trabajadores de Comercio Justo (CLAC) (2016), el asunto en cuestión, es el uso de productos sintéticos, porque con estos, no solo mueren las plagas que afectan el cultivo, sino también, otros animales y plantas que hacen su aporte al ecosistema. Por lo general, los plaguicidas son considerados como la fórmula más directa entre "costo y efectividad para controlar las plagas sean hierbas o insectos. Sin embargo, su movilidad en el ambiente y su toxicidad los hacen sustancias peligrosas que pueden llegar a contaminar suelos, aire y agua" (Morales, 2013, p. 36).

En el CBMA se identifica una mejoría en cuanto a la técnica de siembra, pero existen otros elementos importantes que llevan a un detrimento del ambiente por la generación de acciones contaminantes y que puede conllevar a la reducción de las especies. Ejemplo de ello, el uso del mismo equipo para esparcir plaguicidas y abonar las plantas; o bien, el lavarlo de este mobiliario en fuentes de agua. Es importante tener presente que los plaguicidas pueden tener niveles de toxicidad que no solo afecta la biodiversidad silvestre sino también a las personas, causando "efectos crónicos como trastornos neurológicos, cáncer, alteraciones oftalmológicas, neumonitis y fibrosis pulmonar, trastornos del sistema inmunitario, lesiones hepáticas y cistitis hemorrágica, entre otros" (Morales, 2013, p. 30). Retomando el planteamiento de González (2018) es necesario evaluar las prácticas y capacitar a los agricultores; indagar cuáles son las acciones que persisten en detrimento del ambiente y la salud humana; develar las estrategias que deben aplicarse en estos procesos para generar producción que no contamine y a la vez se apoye; de manera que no se genere "un conflicto de contenido ambiental, ya que estos se producen cuando se tensiona la estabilidad entre una comunidad y su hábitat (Montero \& Sandí, 2008, 31). Se puede afirmar que "el desafío para las personas que gestionan la tierra radica en tener que diseñar e implementar estrategias que garanticen la conservación y migración (movilidad) de la biodiversidad frente a las demandas que compiten por dicha utilización" (Schlönvoigt, 2012, p. 15). Por tanto, se deben formar en prácticas eco-sostenibles que sean armoniosas con el ambiente y la vida en todas sus manifestaciones. Por ejemplo, el uso de plaguicidas y herbicidas orgánicos, lo cual ayude a controlar las plagas, se generan beneficios sociales y la viabilidad económica tan necesaria para la subsistencia (CLAC, 2016).

Con el cultivo de café puede generarse acciones que armonizan con el ambiente si se acompaña con las técnicas adecuadas. De acuerdo con Vignola, Watler, Poveda y Vargas (2018) el café contribuye en reducir la radiación, mejorar el balance hídrico y aumentar la humedad relativa dentro del cafetal, entre otros aspectos. Esta acción debe aprovecharse por los entes involucrados para brindar instrucción sobre la relación de mutualismo entre las especies; de manera tal, que se cultive el café con árboles, que además de brindar sombra al cultivo, den fruto y sirvan de alimento a las diversas especies de la zona; lo cual contribuye con el resguardo de la biodiversidad.

Continuando con el análisis, el 26\% del territorio del CBMA corresponde a repasto (SINAC 2015). La actividad ganadera genera "la corta de muchos árboles para pasto" (E 96). Además, el 5\% de los encuestados considera que existen pastizales sin árboles dispersos lo que agrava la emisión de gases en la atmósfera por parte del ganado.

De acuerdo con el Ministerio de Agricultura y Ganadería (2018) en la ruta hacia la descarbonización de la ganadería en Costa Rica, el $48.9 \%$ de las emisiones se relaciona con la actividad agropecuaria; de estas, el $41.5 \%$ es originada por la ganadería. De las emisiones totales del país, un 20,3\% corresponde al ganado propiamente. No obstante, la ganadería es una de las actividades más importantes de la economía costarricense, y en este sentido, existen esfuerzos importantes por parte de entes gubernamentales y de la sociedad civil orientados a desarrollar prácticas en pro de un ambiente saludable. Este accionar se reafirma en el informe Estrategia para la Ganadería Baja en Carbono en Costa Rica (2015), donde se plantea la meta orientada a la descarbonización del país en procura del desarrollo sostenible, el cual se hará efectivo cuando se genere un equilibrio entre la explotación de los recursos y su recuperación natural (Zarta, 2018). 
Con miras a la descarbonización del país, debe promoverse acciones que contribuyan con oxigenar el planeta. Al respecto, es necesario generar propuestas de capacitación, que permita comprender no solo los beneficios que brinda la reforestación en la producción ganadera sino también a los ecosistemas; ya que esta, representa suplemento alimenticio para el ganado, sirve para cosechar madera, brinda sombra, les provee refugio y permite el desplazamiento de los animales silvestres. Así mismo, es fundamental reforestar con especies que no afecten el pastizal y se contribuya con el secuestro de carbono y fijación del nitrógeno en las fincas (Sánchez, 2017). Es importante promover prácticas sostenibles como delimitar las fincas y los espacios con cercas vivas, lo que genera un ahorro económico y regeneración del entorno. Otro aspecto clave, es incursionar en la generación de abono orgánico a partir de los desechos del ganado. De acuerdo con la Organización de las Naciones Unidas para la Alimentación y la Agricultura, se pueden generar productos de composteo no contaminantes para esparcir en las fincas lo cual contribuye en reducir los gases generadores del efecto invernadero (Román, Martínez \& Pantoja, 2013).

En cuanto al cambio en la estructura del paisaje en la zona del corredor biológico, este se genera principalmente con la corta de árboles para dar paso a las actividades productivas y a la creación de urbanizaciones, carreteras y locales comerciales. Esta situación pone en riesgo la conectividad estructural y funcional del corredor biológico mediante "la expansión urbana, la pérdida y la degradación de espacios verdes" (Potthast \& Geppert, 2012, p. 9). Por tanto, el éxito en preservar la biodiversidad en los corredores biológicos "depende en gran medida de la capacidad de la fauna y flora de sobrevivir en paisajes fragmentados y con predominio de la presencia humana" (Schlönvoigt, 2012, p. 15). El panorama descrito evidencia la situación crítica; si las poblaciones humanas que habitan el corredor biológico no priorizan la conservación de los ecosistemas naturales, la biodiversidad natural saldrá afecta irremediablemente.

Si bien, el café y la ganadería movilizan la economía del CBMA, estas actividades productivas generan colateralmente otros problemas, por lo que es necesario promover el desarrollo desde una óptica sostenible tal y como lo plantea Peter Nijkamp, una relación equilibrada entre el crecimiento económico, la equidad social y la sustentabilidad ambiental para dar lugar al desarrollo sustentable (Zarta, 2018). En esta línea, ante los problemas socio-ambientales es necesario su análisis desde la concepción del modelo de desarrollo que se promueve, donde se asuma el ámbito social y ambiental de manera paralela con el desarrollo económico (Morales, Aliste, Neira \& Urquiza, 2019).

En el corredor biológico existe la percepción que el cuido del agua depende de las Asadas y no se establece relación directa con las prácticas productivas que pueden alterar la calidad de esta. Defender el agua no es solo que sea administrada por la comunidad o creer que la calidad depende de quien la administra, se debe comprender que este es un bien común, responsabilidad de todos y las acciones que se desarrollan en el entorno natural la afecta directamente.

Se destaca que las personas reconocen que no aplican prácticas en favor del ambiente (49\%), persiste la deforestación, la contaminación y la reducción de la biodiversidad (43\%); así como la percepción, que existe el discurso de cuidar el ambiente, pero las personas realmente no desarrollan acciones concretas que sean coherentes con este enfoque. De acuerdo con la "Encuesta sobre patrones y percepciones ambientales realizada por el Programa del Estado de la Nación en el año 2017, evidenció que la ciudadanía está lejos de presentar comportamientos sostenibles en su vida cotidiana (Castro, 2019, p. 20).

Es preciso comprender que el agua es responsabilidad del colectivo social, que el aumento en el consumo, el deterioro de su calidad y la escasez, atentan contra la supervivencia planetaria. De acuerdo con la Organización de las Naciones Unidas (ONU) (2019), existe en el mundo más de 2.000 millones de personas que sufren carencia del agua. La situación del agua en todo el mundo es crítica, la realidad planetaria muestra que cada año se incrementa en un $1 \%$ la demanda mundial (ONU, 2019). La crisis por el agua es real y la protección no puede mirarse de manera reductiva. En el caso de Costa Rica, que cuenta con gran riqueza hídrica, el líquido empieza a escasear (Valverde, 2013). Esta situación no es ajena en el CBMA, se identifica 
"30 familias que carecen de agua potable en sus hogares" (EP-2), lo que debe llevar a reflexionar y generar acciones proactivas para revertir el daño ambiental con visión integral y ecosistémica.

De acuerdo con lo expuesto, se requiere conciencia ambiental, lo cual tiene que ver con "el conocimiento que el ser humano tiene del entorno que le rodea para cuidarlo" (Barón, 2014, p. 43). Como entramado social se debe tener consciencia de los problemas ambientales y la huella ecológica individual y social. El medio ambiente se debe asumir como parte de la responsabilidad social, entendiendo esta como un deber ciudadano con el planeta y las generaciones que lo heredan.

La protección del entorno natural solo puede lograrse mediante agendas comunes entre el Estado, los gobiernos locales y la sociedad civil. De acuerdo con Canet, Finegan y Herrera (2012), los corredores biológicos se constituyen en espacios propicios para la concertación social, donde participan diversos actores con metas ambientales comunes. Precisamente, esta plataforma debe aprovecharse para generar conciencia y acciones en pro del ambiente. Al respecto, se rescata que el 59\% de los encuestados tiene la percepción de contribuir con el ambiente, y se está dando evidencia en asumir la responsabilidad ciudadana con el cierre de dos actividades productivas con afectación al ambiente. Este logro es importante, las comunidades redescubren el poder que tienen como colectivo a partir de la cohesión social y la comprensión que pueden incidir en el desarrollo local con acciones orientadas a la concientización-acción y si es preciso, a la denuncia formal en aras del mejoramiento de la comunidad, el ambiente y mayor justicia social (Mosaiko, s.f.).

Como parte de la organización local, se identifica en el CBMA la incursión turística aprovechando la riqueza biológica de la zona. Desde la perspectiva de desarrollo sostenible se debe buscar el crecimiento económico con la promoción de bienes y servicios, en correspondencia con el potencial y necesidades comunitarias desde una enfoque local y territorial (Carvajal, 2011), donde la relación hombre-naturaleza minimice los impactos y a la vez, promueva la economía local (Zarta, 2018). Desde este matiz, se identifica en el CBMA un modelo de desarrollo local endógeno donde la iniciativa privada tiene un papel fundamental que se impulsa a partir de la colaboración empresarial y el involucramiento social. Desde este modelo, el desarrollo se orienta en incrementar el bienestar de la comunidad mediante el establecimiento de actividades económicas y socio-ambientales, utilizando mayormente los propios recursos humanos y materiales (Monge \& Masías, 2016).

Desde la perspectiva expuesta, es importante incluir toda iniciativa de desarrollo local en el marco de la Ley para el Fomento del Turismo Rural Comunitario (Ley $N^{\circ}$ 8724); la cual tenga proyección a la sostenibilidad ambiental, de manera que persista el dinamismo económico, social y equilibrio ecológico sin detrimento de los ecosistemas.

De acuerdo con el Informe del Estado de la Nación en Desarrollo Humano Sostenible (2018), a pesar que Costa Rica ha generado valiosos esfuerzos para el resguardo de los recursos naturales, el deterioro y uso irracional de los mismos ha persistido. Se agrega, aunque existe un amplio marco legal, persiste ausencia en la aplicación de la ley ante acciones en menoscabo del ambiente, lo que genera malestar y poca credibilidad. Así mismo, la situación se agrava cuando quienes residen en áreas protegidas desconocen de estas, como es el caso del 93\% de las personas encuestadas, que no tenían referencia de vivir en el entorno del CBMA. Así mismo, un 46,8\% de los encuestados no percibe cambios que atenten contra el ambiente.

De acuerdo con Cambrollé, Muñoz, Luque y Figueroa (2012) la falta de conocimiento se convierte en una amenaza para la conservación de los ecosistemas. El cuido del ambiente requiere personas capacitadas ya que esto "facilita la comprensión de los procesos ambientales en relación con los sociales, económicos y culturales, de manera política" (Martínez, 2010, p.101).

Una ciudadanía instruida puede generar resistencia, debate y reivindicación en pro de los recursos naturales, así como acciones colectivas para mitigar los daños al ambiente. Por tanto, el problema radica, que si no se tiene conocimiento y consciencia de las acciones que deterioran el ambiente, difícilmente podrán analizar las propias prácticas o desarrollar cambios en favor de la sostenibilidad ambiental de manera individual. 
Los impactos humanos en los ecosistemas de los CB, devela la necesidad de formar a la ciudadanía en cultura ambiental sostenible como una forma armoniosa para relacionarnos con el entorno; interacción que debe estar mediada por valores y actitudes que impregnen un comportamiento tendiente a la protección de los recursos naturales (Miranda, 2013). En este sentido, la educación ambiental debe proveer un marco de referencia para el análisis de la interacción de la sociedad con el ambiente, de manera que se reconstruyan aprendizajes y se genere consciencia ciudadana para establecer mejores relaciones y conductas en favor del medio ambiente (Martínez 2010, p. 113), ya que el desarrollo sostenible se logrará en la medida que la educación ambiental trasverse todas las áreas de la sociedad, para lo cual se requiere la intencionalidad política, de manera que se forme en estilos de vida eco-sostenibles, como capital cultural para ser heredado.

\section{Consideraciones Finales}

El estudio que se desarrolla a partir de las percepción y prácticas de las comunidades que habitan el corredor biológico, brinda un marco de referencia sobre el estado situacional del CBMA. Las percepciones en conjunto con otras habilidades cognitivas contribuyen en formar pensamiento, y este a su vez, prácticas concatenadas que se derivan de la forma de pensar (Álvarez, 2010). Las percepciones planteadas, son un proceso activo-constructivo que no queda en el plano de la recepción de información; al contrario, estas intervienen en la interpretación de la realidad (Neisser, 1967; Coll, 1987). Por tanto, las percepciones de las personas que habitan el CBMA contribuye no solo en brindar insumos para monitorear y detectar modificaciones que se van dando en el territorio, sino que sustentan en alguna medida las conductas que las personas desarrollan cotidianamente.

En el estudio socio-ambiental se identifica contaminación por desechos sólidos, por lo que es necesario promover propuestas de capacitación sobre lo que implica el proceso de reciclaje, donde es necesario posicionar el énfasis en la reducción de productos contaminantes y prácticas que minimicen los desechos sólidos en los hogares. Así mismo, es necesario promover la importancia de la regeneración y protección de los bosques por parte del colectivo social.

Desde la perspectiva de desarrollo sostenible del territorio, se requiere en el CBMA una planificación estructural, de manera que no aumente la fragmentación de los ecosistemas y se promueva el progreso local de manera estratégica y ecosostenible, de modo que el turismo rural no afecte el entorno natural y se movilice la economía comunitaria.

Para finalizar, se recomienda estudios que profundicen en el cambio de las condiciones climáticas en algunos sectores del territorio del corredor biológico, ante la merma de agua y la preocupación de vivenciar sequía (zona de Zapotal). Así mismo, se requiere procesos investigativos sobre el estado situacional de algunos insectos polinizadores, ya que en varias comunidades no se reportan especies como la abeja melipona. Se sugiere fortalecer acciones orientadas en brindar conocimiento y concientización para el desarrollo de acciones en torno a la sostenibilidad ambiental, como parte de la responsabilidad ciudadana en heredar un mejor planeta.

\section{Agradecimiento}

El estudio se realizó con el apoyo del Fondo Institucional de Desarrollo Académico (FIDA) y la División de Educación Rural, de la Universidad Nacional de Costa Rica (Código presupuestario 059949-0000).

\section{Referencias}

Aliste E. y Rabi V. (2012). Concebir lo socio-ambiental: representación y representatividad en los discursos sobre el desarrollo. Polis. 11 (32). https://www.redalyc.org/pdf/305/30524549015.pdf 
Armenteras, D., González, T., Vergara L., Luque, F., Rodríguez, N. y Bonilla, M. (2016). Revisión del concepto de ecosistema como "unidad de la naturaleza" 80 años después de su formulación. Ecosistemas, 25(1), 83-89. 10.7818/ECOS.2016.25-1.12

Asamblea Legislativa de la República de Costa Rica. Decreto Ejecutivo No 33106-MINAE. 9 de febrero de 2010. La Gaceta No 103 , San José. CR. 30. 3-4. http://www.pgrweb.go.cr/scij/Busqueda/Normativa/Normas/nrm_texto_completo.aspx?nValor1=1\&nValor2=57278

Asamblea Legislativa de la República de Costa Rica. Política Nacional de Biodiversidad $2015-2030.1^{\circ}$ de julio de 2019. http://www.pgrweb.go.cr/scij/Busqueda/Normativa/Normas/nrm_texto_completo.aspx?param1=NRTC\&nValor1=1\&nValor2=89370\&nValor3=117296\&strT ipM=TC

Asamblea Legislativa de la República de Costa Rica. Reglamento a la Ley de Biodiversidad. Decreto № 34433 . La Gaceta Nº 68 del 8 de abril del 2008. Poder Ejecutivo -MINAE. https://www.conagebio.go.cr/Conagebio/public/documentos/legislacion/Decretos/Reglamento_a_la_Ley_Biodiversidad_DE_34433MINAE.pdf

Barón, G. (2014). El medio ambiente es responsabilidad social de todos Facultad de Psicología. (Trabajo de Grado. Universidad Católica de Colombia). https://repository.ucatolica.edu.co/bitstream/10983/1711/1/El\%20medio\%20ambiente\%20es\%20responsabilidad\%20social\%20de\%20todos\%20\%20Facultad $\% 20 \mathrm{de} \% 20$ Psicologia\%20Universidad\%20Cat\%C3\%B3lica.pdf

Bennett, A. (2003). Linkages in the Landscape: The Role of Corridors and Connectivity in Wildlife Conservation. IUCN, Gland, Switzerland and Cambridge, UK. https://portals.iucn.org/library/efiles/documents/FR-021.pdf

Calderón, E. (2019). Participación ciudadana en la gestión sostenible de la biodiversidad. Ambientico, $272 . \quad 28-34$. http://www.ambientico.una.ac.cr/pdfs/ambientico/272.pdf

Cambrollé J., Muñóz S., Luque T. y Figueroa, M. (2012). La amenaza del desconocimiento en la conservación de la diversidad vegetal europea: El caso de la adormidera marítima. Ecosistemas. 21(3), 110-112. 10.7818/ECOS.2012.21-3.16

Canet, L., Finegan, B. y Herrera, B. (2012). Efectividad de manejo en corredores biológicos: el caso de Costa Rica. Parques, (2), 2-11.

Carvajal A. (2011). Desarrollo Local. Manual Básico para Agentes de Desarrollo Local y otros actores. Universidad del Valle. 235 p. Recuperado el 13 octubre de 2020 de: eumed.net

Castro, M. (2019). Área de Conservación Central: promoviendo conectividad estructural y funcional através de corredores biológicos interurbanos en Costa Rica. Ambientico 272. 5-12. http://www.ambientico.una.ac.cr/pdfs/ambientico/272.pdf

Coll, C. (1987). Psicología y currículum. Paidós. Barcelona, España. Coordinadora Latinoamericana y del Caribe de Pequeños Productores y Trabajadores de Comercio Justo (2016). Manual de fertilizantes y plaguicidas orgánicos en el cultivo de café.http://clac-comerciojusto.org/wpcontent/uploads/2015/04/manual-plaguicidas-org\%C3\%A1nicos.pdf

Cuenca, P., Guevara, J. y Franco W. (2018). Ecosistemas, Biodiversidad y Conservación. IKIAM. Napo. EC. 162 p. https://www.researchgate.net/publicatio n/33932 578 0_Ecosistemas_Biodiversidad_y_Conservacion

Fernández C., Hernández R., y Baptista, M. (2014). Metodología de la Investigación. Mac Graw Hill. https://www.uca.ac.cr/wpcontent/uploads/2017/10/Investigacion.pdf $\quad$ https://www.ecolex.org/details/legislation/ley-no-8724-ley-de-fomento-del-turismo-rural-comunitario-lexfaoc089985/

González, T. (2018). Los pequeños productores de café en Chiapas y el desarrollo de capacidades locales a partir del proceso de integración al comercio justo. (Trabajo de posgrado, Colegio de la Frontera) https://www.colef.mx/posgrado/wp-content/uploads/2018/10/TESIS-Gonz\%C3\%A1lez-Escalante-Teresa.pdf

Haddis, A. y Devi, R. (2008). Effect of effluent generated from coffee processing plant on the water bodies and human health in its vicinity. Journal of Hazardous Materials. 152, 259-262.

Instituto del Café de Costa Rica (2017). Informe sobre la actividad cafetalera de Costa Rica. ICAFE. http://www.icafe.cr/wpcontent/uploads/informacion_mercado/informes_actividad/anteriores/2017.pdf

Ley Fomento del Turismo Rural Comunitario. Decreto № 8.72417 de julio del 2009, publicada en La Gaceta No.191 del 01 de octubre del 2009

Martínez, R. (2010). La importancia de la educación ambiental ante la problemática actual. Educare, XIV (1). 97-111. https://doi.org/10.15359/ree.14-1.9

Ministerio de Agricultura y Ganadería (2015). Estrategia para la Ganadería Baja en Carbono en Costa Rica. MAG. http://www.mag.go.cr/bibliotecavirtual/L01-11006.pdf

Ministerio de Agricultura y Ganadería de Costa Rica (2018). Costa Rica en ruta hacia la descarbonización de la ganadería $2013-2018 . \quad$ MAG. http://www.mag.go.cr/bibliotecavirtual/L01-11104.PDF

Ministerio de Ambiente y Energía (2006). Programa Nacional de Corredores Biológicos. MINAE.

Ministerio de Ambiente, Energía y Telecomunicaciones. Política Nacional de Biodiversidad 2015-2030. MINAE. https://www.conagebio.go.cr/Conagebio/public/documentos/POLITICA-NACIONAL-DE-BIODIVERSIDAD-2015.pdf

Ministerio de Ambiente y Energía (2016). Regulación del Programa Nacional de Corredores Biológicos. Decreto N ${ }^{\circ} 40043$-MINAE. Recuperado de http://www.pgrweb.go.cr/scij/Busqueda/Normativa/Normas/nrm_texto_completo.aspx?param1=NRTC\&nValor1=1\&nValor2=83424\&nValor3=107128\&strT ipM=TC 
Research, Society and Development, v. 10, n. 6, e30410615842, 2021

(CC BY 4.0) | ISSN 2525-3409 | DOI: http://dx.doi.org/10.33448/rsd-v10i6.15842

Ministerio de Ambiente y Energía (2018). Resumen del Sexto Informe Nacional de Costa Rica ante el Convenio de Diversidad Biológica. Programa de Naciones Unidas para el Desarrollo. MINAE.

Miranda, L. (2013). Cultura ambiental: un estudio desde las dimensiones de valor, creencias, actitudes y comportamientos ambientales. Producción + Limpia 8 (2), 94-105.

Monge, Y. y Masías, A. (2016). El desarrollo local. Enfoques para su conceptualización. Olimpia. 13(39), 226-238.

Montero, A. y Sandí, J. (2009). Contaminación de aguas por el beneficiado del café en Costa Rica entre 1840 y 1910. Ciencias Ambientales, 37 (1), $30-35$. http://dx.doi.org/10.15359/rca.37-1.4

Morales, B., Aliste, E., Neira, I. y Urquisa A. (2019). La compleja definición del problema socioambiental: racionalidades y controversias. MAD. $10.5354 / 0719-0527.2019 .54834$

Morales, R. (2013). Metodología de análisis del riesgo por contaminación de agroquímicos: de Salud Pública, 22 (1), 35-44. https://www.scielo.sa.cr/pdf/rcsp/v22n1/art07v22n1.pdf

cuenca del Río San Blas, Costa Rica. Revista Costarricense

Mosaiko (s.f.) Reflexiones sobre Movilización social 2008-2018. https://iniciativasdecooperacionydesarrollo.files.wordpress.com/2012/04/2012 _04_docmovilizacionmosaiko.df

Neisser, U. (1967). Cognitive psychology. New York. EEUU. Appleton-Century-Crofts. Organización de las Naciones Unidas (2019). No dejar a Nadie Atrás: Informe Mundial de las Naciones Unidas sobre el Desarrollo de los Recursos Hídrico. shttps://www.acnur.org/5c93e4c34.pdf

Potthast, M. y Geppert, S. (2012). Corredores Biológicos Interurbanos: Fusionando el capital construido y el capital natural de la ciudad. Ambientico, 272, 512. http://www.ambientico.una.ac.cr/pdfs/ambientico/272.pdf

Programa Estado de la Nación en Desarrollo Humano Sostenible (2018). Informe Estado de la Nación (2018) http://www.asamblea.go.cr/sd/Documents/analisis/Inforne\%20Estado\%20de\%20\%20La\%20Naci\%C3\%B3n\%202018.pdf

Román P., Martínez, M. y Pantoja, A. (2013). Manual de Compostaje del Agricultor. FAO. http://www.fao.org/3/a-i3388s.pdf

Sánchez, L. (2002). II Curso Internacional de Aspectos Geológicos de Protección Ambiental. UNESCO. UY. 322-331

Sánchez, R. (2017). Plan de sostenibilidad para el fortalecimiento de la actividad cafetalera en fincas ubicadas en la comunidad de Rincón de Mora, San Ramón, Alajuela, 2016-2017. (Tesis de Posgrado, Universidad de Costa Rica).https://www.sep.ucr.ac.cr/posgrados/desarrollosostenible/tesis/ronald_sanchez.pdf

Schlönvoigt, M. (2019). Corredores Biológicos Interurbanos: instrumentos de conservación y bienestar. Ambientico, 272. 13-18. http://www.ambientico.una.ac.cr/pdfs/ambientico/272.pdf

Sistema Nacional de Áreas de Conservación (2015). Corredor Biológico Montes del Aguacate: Plan para la Gestión Estratégica 2015-2021. En H. Acevedo y. Villalobos (Eds.), Área de Conservación Cordillera Volcánica Central. San Ramón-Costa Rica. MINAE. https://conservationcorridor.org/cpb/SINAC_2015.pdf

Sistema Nacional de Áreas de Conservación (2018). Plan Estratégico 2018-2025: Programa Nacional de Corredores Biológicos de Costa Rica. MINAE.

Solano, F. (2017). Propuesta de zonificación ambiental del corredor biológico interurbano Río María Aguilar. Revista de Ciencias Ambientales, 51(1), 33-50. http://dx.doi.org/10.15359/rca.51-1.3

Universidad Nacional (2018). Análisis de los Corredores Biológicos como estrategia para la conservación y el desarrollo local en Costa Rica (código 015617). DER. Heredia, CR. Autor.

Valverde, R. (2013). Disponibilidad, distribución, calidad y perspectivas del agua en Costa Rica. Ambientales (45). http://www.ambientico.una.ac.cr/pdfs/ambientales/45.pdf

Vargas L. (1994). Sobre el concepto de percepción. Alteridades, 4 (8), 47-53 https://www.redalyc.org/pdf/747/74711353004.pdf

Vignola, R., Watler, R., Poveda K. y Vargas, A. (2018). Prácticas efectivas para la reducción de impactos por eventos climáticos en el cultivo de café en Costa Rica. CATIE. http://www.mag.go.cr/bibliotecavirtual/reduccion-impacto-por-eventos-climaticos/Informe-final-cafe.pdf

Zarta, P. (2018). La sustentabilidad o sostenibilidad: un concepto poderoso para la humanidad. Tabula Rasa (28), 409-423. http://dx.doi.org/10.25058/20112742.n28.18. 